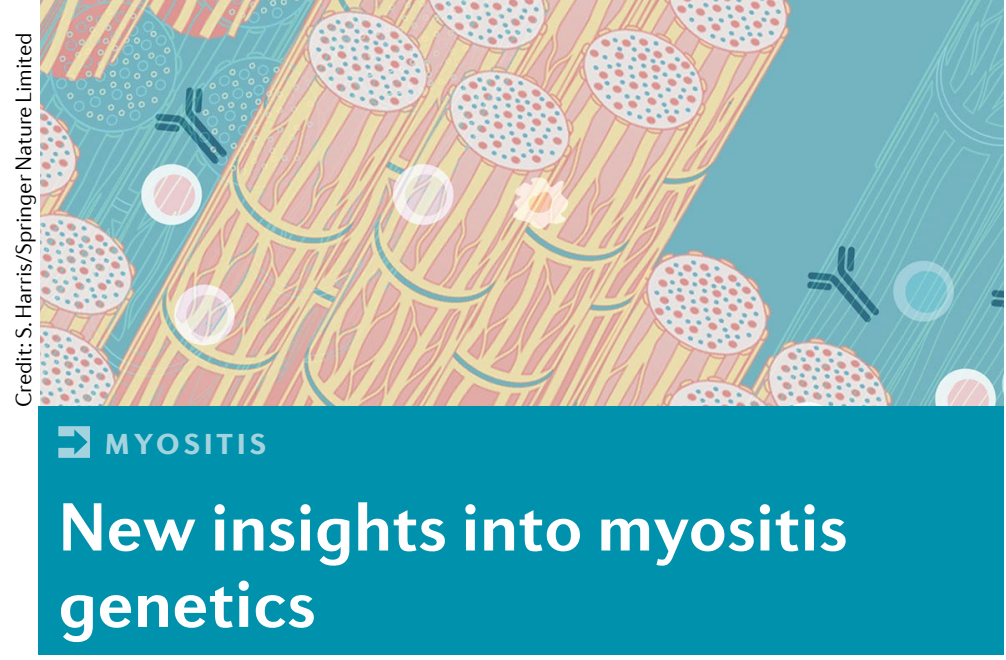

A large international study investigating genetic risk factors in autoantibody-defined subgroups of patients with idiopathic inflammatory myopathy (IIM; also known as myositis) has revealed novel strong associations with amino acid positions within HLA molecules. The research also revealed genetic differences between juvenile-onset and adult-onset disease in patients with the same autoantibody specificity.

"Traditionally, the strongest genetic risk factors for IIM autoantibodies have been described with classical HLA alleles," explains corresponding author Simon
Rothwell. "In this study, we have refined these associations by identifying specific amino acid positions within HLA molecules that may confer risk, providing mechanistic insights into IIM."

Rothwell and colleagues analysed genetic data from 2,582 patients enrolled in the Myogen Genetics Consortium, who were divided into 12 subgroups according to the presence of myositis-relevant antibodies. As expected, strong associations with HLA alleles were found within the autoantibody-defined subgroups. For some autoantibodies, associations with amino acid positions were as strong, or stronger, than the classical HLA associations.

Notably, in patients with anti-TIF1 autoantibodies, associations with HLA-DQB1 alleles differed between those with adult-onset IIM and those with pediatric-onset IIM. "The presence of anti-TIF1 autoantibodies is strongly associated with cancer in adult-onset IIM but not in juvenileonset IIM," Rothwell highlights. "The identification of independent genetic risk factors suggests distinct aetiologies and disease mechanisms in these patients."

The analysis provides new insights into the functional importance of genetic risk factors for IIM. "As autoantibodies in IIM correlate with clinical features of disease, understanding genetic risk underlying the development of certain autoantibody profiles has implications for understanding disease progression and prognosis," concludes Rothwell.

Sarah Onuora with pediatriconset IIM

ORIGINAL ARTICLE Rothwell, S. et al. Focused HLA analysis in Caucasians with myositis identifies significant associations with autoantibody subgroups. Ann. Rheum. Dis. 78, 996-1002 (2019)

\section{Origins of synovial macrophages revealed}

Synovial macrophages (SMs) are one of the two main cell types that make up the synovial membrane. Although the origins of tissue-resident macrophages are known for some organs, the exact origin of SMs has been a mystery.

“Previous SM-related studies implied that SMs have two major origins: resident cells and circulating cells," explains corresponding author Wei Wei. "However, owing to the limited number and inaccessibility of SMs, no systematic analysis of SM ontogeny in either physiological or pathological conditions has been performed until now." In their new study, Wei and colleagues used CX3CR1+/GFP mice, which express green fluorescent protein in $\mathrm{CX} 3 \mathrm{CR} 1^{+}$cells, to track the emergence of SMs through several embryonic stages, and bone marrow chimeras to track these cells in adult mice. Two types of SM were found: embryonic SMs (ESMs), which were $\mathrm{F} 4 / 80^{+} \mathrm{CD} 11 \mathrm{~b}^{-}$and appeared at a mid-embryonic stage; and bone marrow-derived SMs (BMSMs), which were $\mathrm{F} 4 / 80^{-} \mathrm{CD} 11 \mathrm{~b}^{+}$and appeared at a late-embryonic stage.

Both ESMs and BMSMs were present in adult mice, but ESMs predominated owing to a post-natal burst in proliferation. The two types of cells also had different phenotypes:

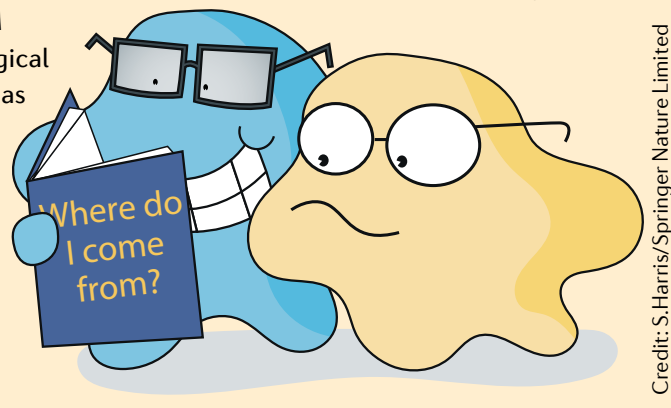

ESMs expressed antiinflammatory cytokines ... and BMSMs expressed proinflammatory cytokines

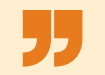

ESMs expressed anti-inflammatory cytokines such as IL-4 and IL-10, and BMSMs expressed pro-inflammatory cytokines such as IL- $1 \beta$ and TNF.

In mice with collagen-induced arthritis, the number of ESMs decreased during disease development and then increased during resolution, whereas BMSMs did the opposite, suggesting a pathologic role for BMSMs. Both types of SM (defined by expression of CD11b and EMR1, the human homolog of $\mathrm{F} 4 / 80$ ) were also present in synovium from patients with rheumatoid arthritis (RA) and had similar anti-inflammatory and pro-inflammatory phenotypes to the equivalent cells in mice.

"Macrophage-targeted therapy for RA has not had promising results so far; however, the identification of the different origins and functions of SMs may provide novel ideas and direction in this field," concludes Wei.

\section{Joanna Collison}

ORIGINAL ARTICLE Tu, J. et al. Ontogeny of synovial macrophages and the roles of synovial macrophages from different origins in arthritis. Front. Immunol. 10, 1146 (2019) 